\title{
Superconducting single-photon detectors integrated with diamond nanophotonic circuits
}

\author{
Patrik Rath $^{1}$, Oliver Kahl ${ }^{1}$, Simone Ferrari ${ }^{1}$, Fabian Sproll ${ }^{2}$, Georgia Lewes-Malandrakis ${ }^{3}$, Dietmar Brink ${ }^{3}$, \\ Konstantin Ilin $^{2}$, Michael Siegel ${ }^{2}$, Christoph Nebel $^{3}$ and Wolfram Pernice ${ }^{1}$
}

Photonic quantum technologies hold promise to repeat the success of integrated nanophotonic circuits in non-classical applications. Using linear optical elements, quantum optical computations can be performed with integrated optical circuits and can therefore overcome the existing limitations in terms of scalability. In addition to passive optical devices for realizing photonic quantum gates, active elements, such as single-photon sources and single-photon detectors, are essential ingredients for future optical quantum circuits. Material systems that allow for the monolithic integration of all components are particularly attractive, including III-V semiconductors, silicon and diamond. Here, we demonstrate nanophotonic integrated circuits made from high-quality polycrystalline diamond thin films in combination with on-chip single-photon detectors. By using superconducting nanowires that are coupled evanescently to traveling waves, we achieve high detection efficiencies of up to $66 \%$ as well as low dark count rates and a timing resolution of 190 ps. Our devices are fully scalable and hold promise for functional diamond photonic quantum devices. Light: Science \& Applications (2015) 4, e338; doi:10.1038/lsa.2015.111; published online 9 October 2015

Keywords: diamond nanophotonics; integrated optics; superconducting single-photon detectors

\section{INTRODUCTION}

The advent of integrated optical circuits has led to a surge of applications in telecommunications, optical signal processing, and on-chip sensing ${ }^{1-4}$. Combining individually optimized photonic components into complex systems provides a flexible framework for the realization of compact and powerful devices. Although such circuits are predominantly investigated in classical optics, nanophotonic devices hold further promise for emerging applications in quantum optics and photonic quantum technologies ${ }^{5,6}$. Since the first realization that optical quantum computing is possible through the use of linear optical elements, single-photon sources and detectors, the combination of these three essential elements into a single circuit has been a driving goal $^{7-9}$. A particular requirement in this respect is the scalability of all core elements. Although scalability can be readily achieved for linear optical elements through nanofabrication and miniaturization, a scalable implementation of the active parts of the circuits is less straightforward. Here, we demonstrate the scalable realization of single-photon detectors embedded in diamond nanophotonic circuits as a first step toward fully integrated diamond quantum systems.

Single-photon detectors, which are fast and provide high detection efficiency combined with good timing accuracy, are highly sought after for applications in quantum optics, imaging, metrology, and sensing. Depending on the wavelength regime of interest, different technologies can be explored, such as silicon avalanche photodiodes (APDs) for visible wavelengths ${ }^{10}$, photomultiplier tubes, or InGaAsbased APDs for the telecommunication range ${ }^{11}$. In the telecom range,
APDs provide non-perfect performance because of their relatively high dark count rates (DCRs) and their need for gated-mode operation. Furthermore, integration with nanophotonic circuitry is nontrivial. In recent years, superconducting nanowire single-photon detectors (SNSPDs) ${ }^{12-14}$ have been shown to be promising alternatives, particularly when they are integrated directly onto waveguides and into photonic circuits ${ }^{15-17}$. To take advantage of the immense optical bandwidth of SNSPDs ${ }^{18}$, which allows one to detect single photons in the mid-infrared (IR) ${ }^{19}$ and the important near-IR wavelengths $^{20}$ (telecom C-band) and in the visible spectrum ${ }^{21}$, a substrate material for waveguide integrated SNSPDs is needed that is transparent throughout this broad wavelength range. Furthermore, the substrate must be available as a high-quality thin film to support the guided modes in waveguides.

Diamond, with its wide electronic bandgap of $5.47 \mathrm{eV}$, is emerging as a promising material for integrated optics because of its broadband transparency spectrum from $225 \mathrm{~nm}$ into the far IR. Additionally, diamond provides a relatively high refractive index of 2.4 , which allows for the tight confinement of light into subwavelength waveguides. By implementing chemical vapor deposition, diamond thin films on a wafer-scale have become available as a convenient template for nanophotonic circuit fabrication ${ }^{22,23}$. In addition to applications in sensing and optomechanics ${ }^{24-29}$, diamond is of broad interest for quantum optics $^{30-32}$. Although waveguide-integrated SNSPDs have been demonstrated on wide-bandgap silicon nitride ${ }^{16,33}$, realizing integrated single-photon detectors on diamond nanophotonic circuitry

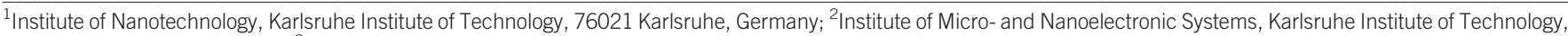
76187 Karlsruhe, Germany and ${ }^{3}$ Fraunhofer Institute for Applied Solid State Physics, Tullastr. 72, 79108 Freiburg, Germany 
is of particular importance because diamond hosts color centers which are suitable for the realization of efficient single-photon sources, such as the nitrogen vacancy center ${ }^{34-37}$ and the silicon vacancy center ${ }^{31,38-40}$.

Here, we present integrated single-photon detectors that are fabricated on top of high-quality polycrystalline diamond nanophotonic waveguides. Using a traveling wave layout, we are able to achieve single-photon detection on-chip with high efficiency. Our detectors have a minimal footprint, which enables us to fabricate hundreds of detector circuits on a single chip. We show that the traveling wave detector can be readily integrated into diamond nanophotonic circuits in a scalable fashion by using established lithography and dry etching procedures. Our results provide a promising route toward all-diamond single-photon circuits for on-chip quantum optics.

\section{MATERIALS AND METHODS}

\section{Detector design and device fabrication}

In order to combine diamond nanophotonic circuits with superconducting single-photon detectors, we fabricate niobium nitride $(\mathrm{NbN})$ nanowires directly on top of diamond waveguides, as shown in the schematic illustration in Figure 1a. The $\mathrm{NbN}$ nanowires are connected to larger metal contact pads, as shown in the scanning electron microscopy (SEM) image in Figure 1b, which allows us to electrically connect the nanowires to a bias current source and appropriate readout electronics. In this way, single photons that are absorbed in the $\mathrm{NbN}$ nanowire can be efficiently detected. Due to the small footprint of the devices, hundreds of photonic circuits, each of which is equipped with its own single-photon detector, can be fabricated in one fabrication run on a $15 \mathrm{~mm} \times 15 \mathrm{~mm}$ wafer die, as shown in the microscope image in Figure 1c.

In contrast to earlier implementations of SNSPDs, in which photons impinge onto the $\mathrm{NbN}$ nanowires from the top ${ }^{12}$, a so-called "traveling wave" geometry ${ }^{15}$ is implemented here, which means that photons propagate along a waveguide and travel parallel to the $\mathrm{NbN}$ nanowire. In this design, the propagating photons are coupled evanescently to the superconducting nanowire and are absorbed in the optical near field. Therefore, the absorption length can be arbitrarily increased by extending the wire length, which is in contrast to the classical SNSPD geometry, where photons impinge onto the detector under normal incidence from the optical far field ${ }^{12}$. In the latter case, the absorption length is limited to the thickness of the NbN layer, which is only a few nanometers thick. Traveling wave SNSPDs, by contrast, feature absorption lengths of tens of micrometers, leading to absorption efficiencies that approach $100 \%{ }^{15}$, thus overcoming a crucial limitation of traditional SNSPDs. Our waveguide detectors are embedded in nanophotonic circuits, as shown in Figure 1c. The circuits use focusing grating couplers ${ }^{41,42}$ to launch light into the on-chip waveguides and to extract transmitted light at a second coupling port. The incoming light is split at a 50:50 beam splitter for calibrating the light intensity inside the waveguide. Light propagating along a second path is guided to the detector, which is attached to the waveguide that leads upwards, away from the grating couplers.

To achieve diamond-based photonic circuitry, diamond thin films surrounded by a cladding material with lower refractive index are required. For this purpose, a $1-\mu \mathrm{m}$-thick polycrystalline diamond film is deposited via plasma-enhanced chemical vapor deposition
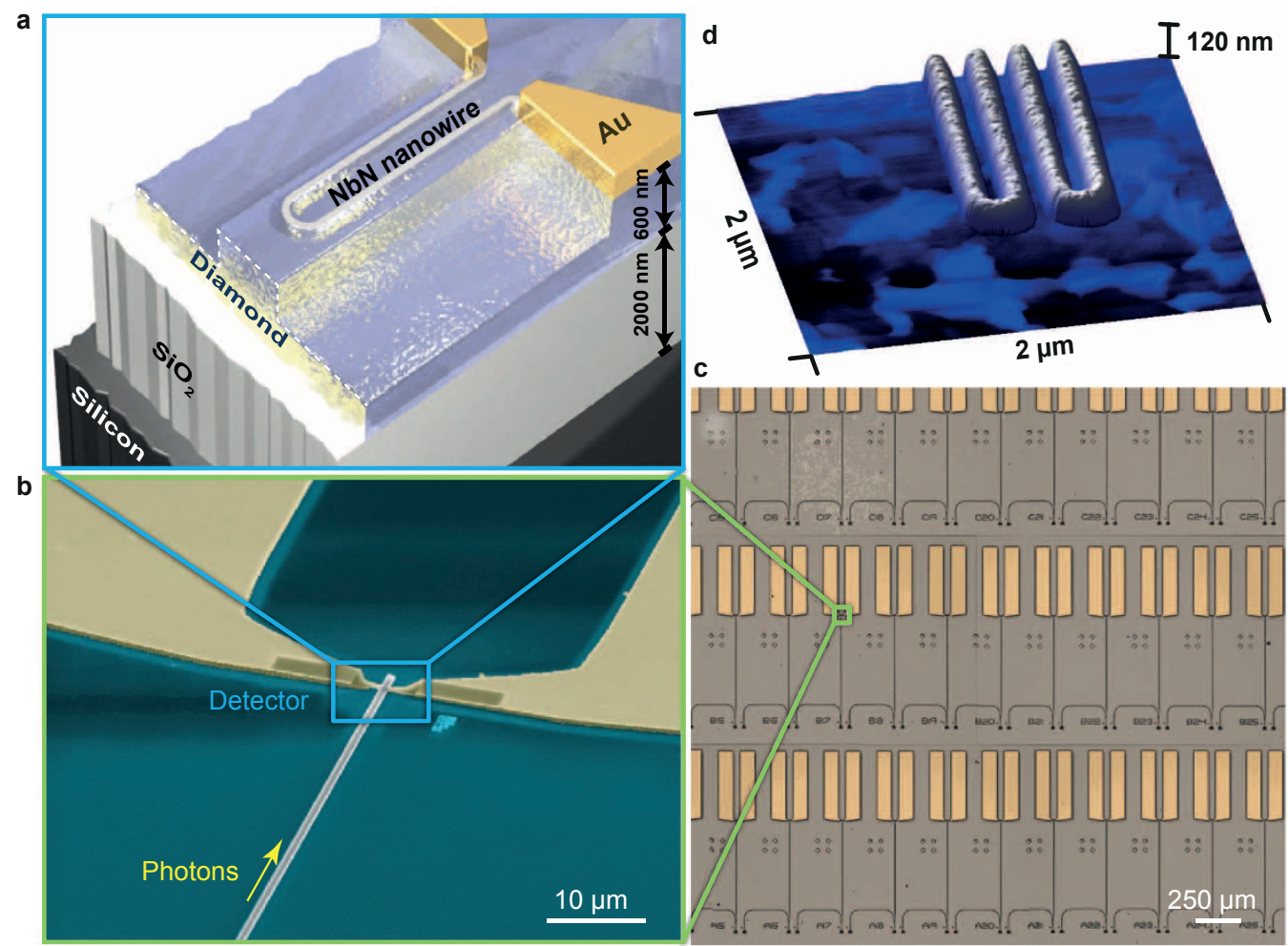

Figure 1 Diamond nanophotonic circuits containing superconducting nanowire detectors. (a) Schematic of an NbN superconducting nanowire detector on top of a diamond rib waveguide. Gold contact pads enable electrical contact with the detector. (b) False color SEM image of one waveguide integrated detector connected to the gold contact pads. (c) Optical microscope image showing a selection of the 192 detectors which were fabricated per sample. (d) Atomic force microscopy scan over a double meander nanowire after the first lithography step. 
(PECVD $)^{43,44}$ onto an oxidized silicon wafer with $2 \mu \mathrm{m}$ of $\mathrm{SiO}_{2}$ on top of a silicon carrier wafer, which results in a wafer-scale diamond-oninsulator (DOI) template ${ }^{22,28}$. Subsequently, the diamond layer is polished to a thickness of $600 \mathrm{~nm}$ by chemo-mechanical polishing with a soft cloth ${ }^{45-47}$ to reduce the root mean square surface roughness to below $3 \mathrm{~nm}^{46}$. Finally, a NbN thin film is sputter-deposited onto the DOI wafer using DC reactive magnetron sputtering in an argon and nitrogen gas mixture. The resulting $\mathrm{NbN}$ layer has a thickness of 4.6 $\mathrm{nm}$ and a critical temperature of $6.5 \mathrm{~K}$, which is well above the base temperature of $1.8 \mathrm{~K}$ of the cryostat used in this work. Although the diamond thin films used in this work are polycrystalline, the fabrication and design routines are general. Therefore, transferring our approach to single crystal DOI substrates is possible. Recent progress in realizing single crystalline DOI templates via transfer techniques provides promising steps in this direction ${ }^{48-50}$. For compatibility with our wafer-scale fabrication approach, however, we restrict our current work to high-quality microcrystalline diamond thin films.

The photonic circuits and the waveguide-integrated SNSPDs are fabricated using three steps of electron beam lithography (EBL) with a JEOL 5500 system (JEOL Ltd, Akishima, Tokyo, Japan) at $50 \mathrm{kV}$ acceleration voltage and subsequent dry etching steps. During the first EBL step, electrodes and alignment markers are written into PMMA positive tone resist. Then, $5 \mathrm{~nm}$ of chromium, $150 \mathrm{~nm}$ of gold, and 10 $\mathrm{nm}$ of chromium are deposited via electron beam physical vapor deposition (PVD) under ultra-high vacuum conditions. A subsequent lift-off step in acetone finalizes the metal structures. A 5-nm-thick glass layer is then sputtered onto the $\mathrm{NbN}$ film in order to promote adhesion of EBL resist. Negative tone resist hydrogen silsesquioxane (HSQ) 6\% is spin coated with a thickness of $120 \mathrm{~nm}$ and structured via EBL to define the nanowires and connection pads to the gold electrodes. This allows us to write thin wires with widths less than $100 \mathrm{~nm}$ while providing a robust etch mask during dry etching using argon plasma to remove the glass adhesion layer and $\mathrm{CF}_{4}$ chemistry to fully etch the $\mathrm{NbN}$ layer. Figure $1 \mathrm{~d}$ shows an atomic force microscopy (AFM) image of such a patterned nanowire, which allows us to quantify the nanowire dimensions and the remaining surface roughness of the DOI wafer.

Next, another glass layer of $5 \mathrm{~nm}$ is sputtered onto the now exposed diamond layer for adhesion promotion during the final EBL step. The photonic structures are defined using 480-nm-thick HSQ 15\% and are transferred into the diamond thin film by reactive ion etching in argon and oxygen plasma ${ }^{47}$. Etching away $300 \mathrm{~nm}$ of the initial diamond layer results in half-etched rib waveguides, as illustrated in Figure 1a. In the resulting overall structure, the $\mathrm{NbN}$ wire is protected from the environment by an HSQ layer, which remains on top of the SNSPD and the waveguide.

\section{Devices for the measurement of the absorption efficiency}

In addition to dedicated single-photon detector circuits, the full chip contains additional photonic circuits to characterize the absorption properties of the $\mathrm{NbN}$ nanowires. The absorption efficiency of light propagating inside the waveguide increases with increasing nanowire width because of a larger waveguide coverage with the superconducting material. For the broadband detection of single-photons, however, narrow nanowires are desirable because the smallest photon energy for which photons can be detected decreases with increasing nanowire width. Therefore, the geometry of the SNSPDs must be optimized for a given wavelength and waveguide mode. In particular, for detecting single photons in the telecommunication range, the nanowire width should be less than $150 \mathrm{~nm}$. To increase the absorption efficiency with narrow nanowires, it is therefore preferable to use a meander layout instead of wider nanowires. Because of the large number of photonic circuits on each chip, we are able to investigate multiple detector device geometries. In particular, two general designs are fabricated and studied in this work, as depicted in Figure 2a: first, a single meander geometry that consists of two parallel straight sections and one connecting bend, and second, a double meander geometry that consists of four parallel straight sections connected in series.

We simulate the guided modes for a $1-\mu \mathrm{m}$-wide half-etched waveguide for a 1550-nm input wavelength with finite element methods using COMSOL Multiphysics. Figure $2 \mathrm{~b}$ shows the mode profiles and corresponding effective refractive indices for a waveguide with a single meander (I and II), with a double meander nanowire (III) and without $\mathrm{NbN}$ nanowire (IV). The waveguide dimensions were intentionally chosen such that the waveguide supports only one transverse electric (TE)-like mode. Due to the large complex refractive index of $\mathrm{NbN}$, the electric field at the nanowire is greatly enhanced, as can be seen in the magnified image (II), which leads to the desired enhanced absorption of photons in the NbN nanowire.

Back reflection of photons at the interface between a bare waveguide and a waveguide with an $\mathrm{NbN}$ wire on top is a possible limiting factor for the detection efficiency of the detector. We estimate the upper bound of this reflection by assuming a sudden change in the effective refractive index of the guided mode between the bare waveguide $\left(n_{\mathrm{eff}}=\right.$ 2.128 ) and the waveguide with a double meander of $100 \mathrm{~nm}$ nanowire width $\left(n_{\text {eff }}=2.130-0.0086 i\right)$, where the imaginary part corresponds to an absorption of $-0.302 \mathrm{~dB} \mu \mathrm{m}^{-1}$. Using Fresnel's equations, we find a reflection coefficient $R=\frac{I_{\mathrm{r}}}{I_{\mathrm{i}}}=4.5 \times 10^{-6}$, where $I_{\mathrm{r}}$ and $I_{\mathrm{i}}$ are the reflected and the incident intensities, respectively. This very low reflection value can be safely neglected in the following analysis.

To experimentally determine the absorption efficiency of the $\mathrm{NbN}$ nanowires on chip, we fabricate dedicated photonic circuits, which allow for balanced optical detection ${ }^{15}$. These devices are fabricated on the same chip and in the same fashion as the detector devices presented in Figure 1. The balanced detection design, as illustrated in Figure 2a, consists of the following components: a focusing grating coupler, as shown in the inset of Figure 2a, that was designed for the efficient coupling of light from a fiber to the TE-like waveguide mode at 1550 $\mathrm{nm}$ is used to launch light into the input waveguide. A Y-Splitter is used as a 50:50 beam splitter: half of the light is guided to the right side and coupled out of the chip via a second grating coupler, which acts as reference port. The other half is guided to the left side, where the propagating mode is attenuated by absorption in the $\mathrm{NbN}$ nanowire and is subsequently coupled out at the "transmission port" grating coupler. Each nanowire is parameterized by a certain wire width and wire length, which are varied from device to device across the chip. This balanced detection design allows us to determine the attenuation due to absorption by the $\mathrm{NbN}$ by dividing the transmitted power $P_{\text {trans }}$ by the power at the reference port $P_{\text {ref. }}$ By using the reference port with identical optical grating couplers and waveguides, the propagation loss and coupling loss do not contribute to the measurement of the absorption coefficient.

\section{RESULTS AND DISCUSSION}

\section{Measurement of the detector absorption efficiency}

The fabricated samples described in the previous section are placed on a fiber-optic measurement setup and aligned against an optical fiber array. Light from a tunable IR laser source (New Focus TLB6600) is coupled into the central waveguide, and the transmitted signals are recorded at both output ports simultaneously. The attenuation due to the nanowire can thus be extracted by calculating the ratio of the 
a

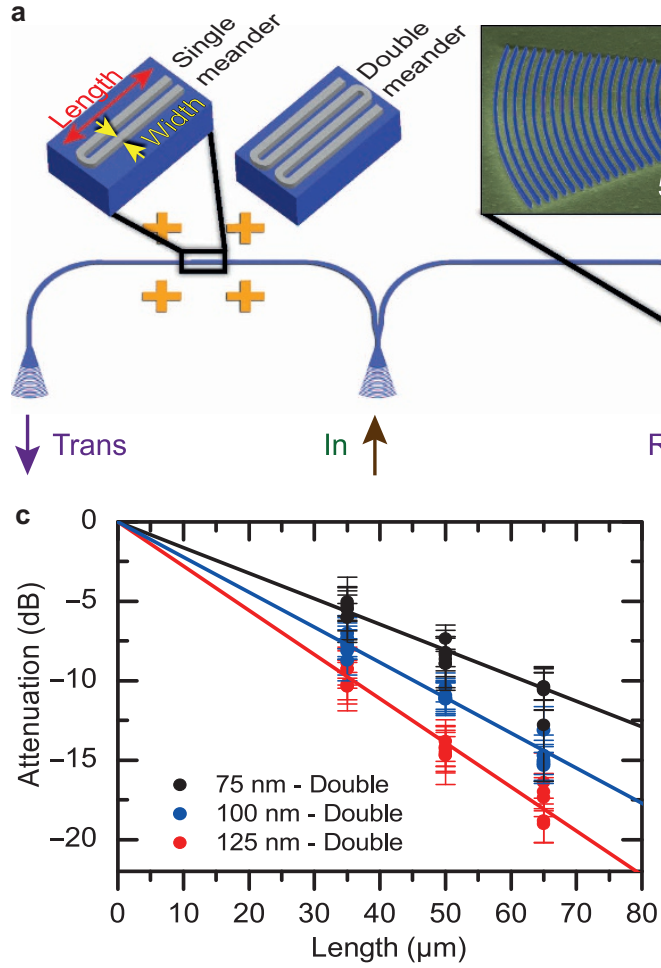

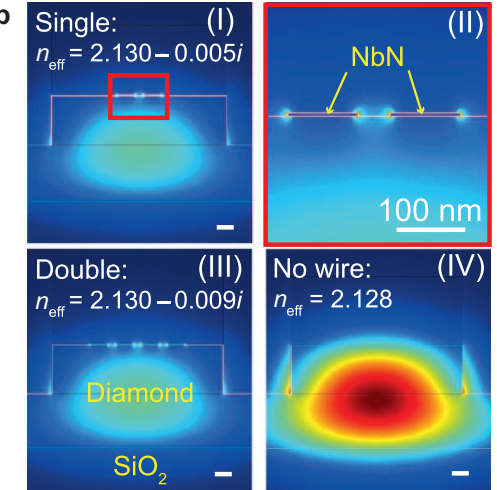

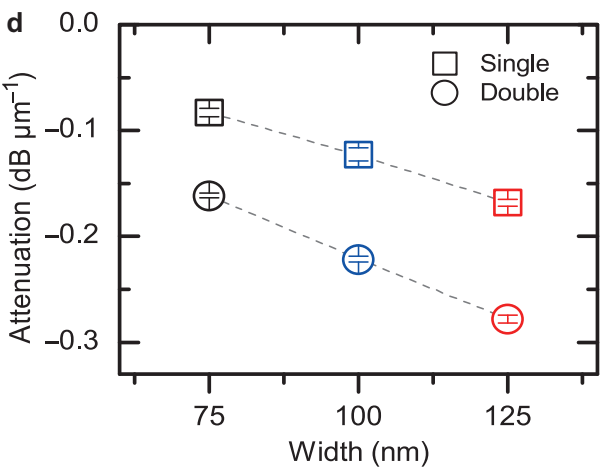

Figure 2 Absorption characteristics of diamond SNSPDs. (a) Schematic of a photonic circuit for balanced detection of the absorption of an NbN nanowire placed on top of the diamond rib waveguide. Inset: false color SEM image of a grating coupler. (b) FEM simulation of the fundamental TE-like optical mode showing the electric field norm for a 1- $\mu \mathrm{m}$-wide rib waveguide with a single meander (I and II), with a double meander (III) and without an NbN nanowire (IV). At the NbN nanowire, the field is greatly enhanced, as observed in the magnified image (II). Scale bars are $100 \mathrm{~nm}$. Subfigure (IV) has a different color scale than the other subfigures to make the distribution of the optical mode in the waveguide more visible. (c) Measured attenuation of light due to absorption by the NbN nanowires with double meander geometry for different widths $(75-125 \mathrm{~nm}$ ) and lengths $(30-70 \mu \mathrm{m})(\mathbf{d})$ Attenuation coefficients extracted from linear fits to the measurement data shown in Figure $2 \mathrm{c}$ for single and double meander nanowires of varying wire widths.

measured transmitted intensities at the transmission and the reference ports. We measure the attenuation for nanowires of three different widths $(75 \mathrm{~nm}, 100 \mathrm{~nm}$, and $125 \mathrm{~nm})$, as shown in Figure 2c, for the double meander geometry. The measurements are initially performed at room temperature. The measured attenuation shows the expected exponential decay with increasing wire length. We extract the attenuation in decibels per micrometer from linear fits to the obtained data for double meanders (Figure 2c) and single meanders (not shown). The fit results, which are depicted in Figure 2d, show the expected attenuation increase with wire width and with the number of parallel $\mathrm{NbN}$ nanowires per waveguide. The largest measured attenuation, for $125-\mathrm{nm}$-wide double meanders, is $-0.278 \mathrm{~dB} \mu \mathrm{m}^{-1}$. This result is slightly smaller than that predicted by the numerical simulations, which we attribute to the fabricated $\mathrm{NbN}$ wire being slightly narrower than the designed width.

\section{Cryogenic measurement of the detector speed}

For detector characterization at cryogenic temperatures, the detector chip is placed into a liquid helium flow cryostat, as illustrated in Figure 3, and cooled to a base temperature of $1.8 \mathrm{~K}$. The chip is mounted on a stack of nano-positioners with closed-loop feedback (Attocube Systems, 80539 Muenchen, Germany) for alignment against the fiber array. The piezo stage allows for controlling the position of the chip in three dimensions and further enables in-plane rotation. At room temperature, the chip is prealigned against the fiber array, and the positions of all of the detector devices are recorded.
After cooling, the photonic circuits are first aligned with respect to the fiber array for light insertion and collection by moving the nanopositioners in the $x / y$-plane (see coordinate system in Figure 3). Then, the RF probe is brought into contact with the gold contact pads of the devices such that the detectors are connected electrically, by moving the nano-positioners in the $z$-direction. The superconducting nanowires are current biased using a low-noise current source and a bias-T acting as a low pass filter. They are then connected to the readout electronics through a second $\mathrm{GHz}$ bias- $\mathrm{T}$ to separate the highfrequency SNSPD signal from the DC bias.

The optical setup consists either of a tunable IR continuous wave laser or a pulsed IR laser combined with two variable optical attenuators and a polarization controller. The on-chip reference port signal is monitored with a lightwave multimeter to enable precise control of the photon flux reaching the SNSPDs with the adjustable optical attenuators, which provide up to $60 \mathrm{~dB}$ attenuation each. The SNSPDs are both biased and read-out via the connected RF probe. The collected signal is then electrically amplified by $85 \mathrm{~dB}$ using low-noise RF amplifiers and is then recorded on a single-photon counting system or a fast oscilloscope. A high signal-to-noise ratio of the photon detection event is achieved by choosing low-noise electrical amplifiers with the appropriate bandwidth, gain, and noise-figure.

Figure 4a shows the average of 500 pulses from a SNSPD measured with a high-speed oscilloscope (blue). The decay time of the pulse is extracted from an exponential fit (red). For a $35-\mu \mathrm{m}$-long double meander detector, the fit yields a decay time of $5.1 \mathrm{~ns}$, corresponding 


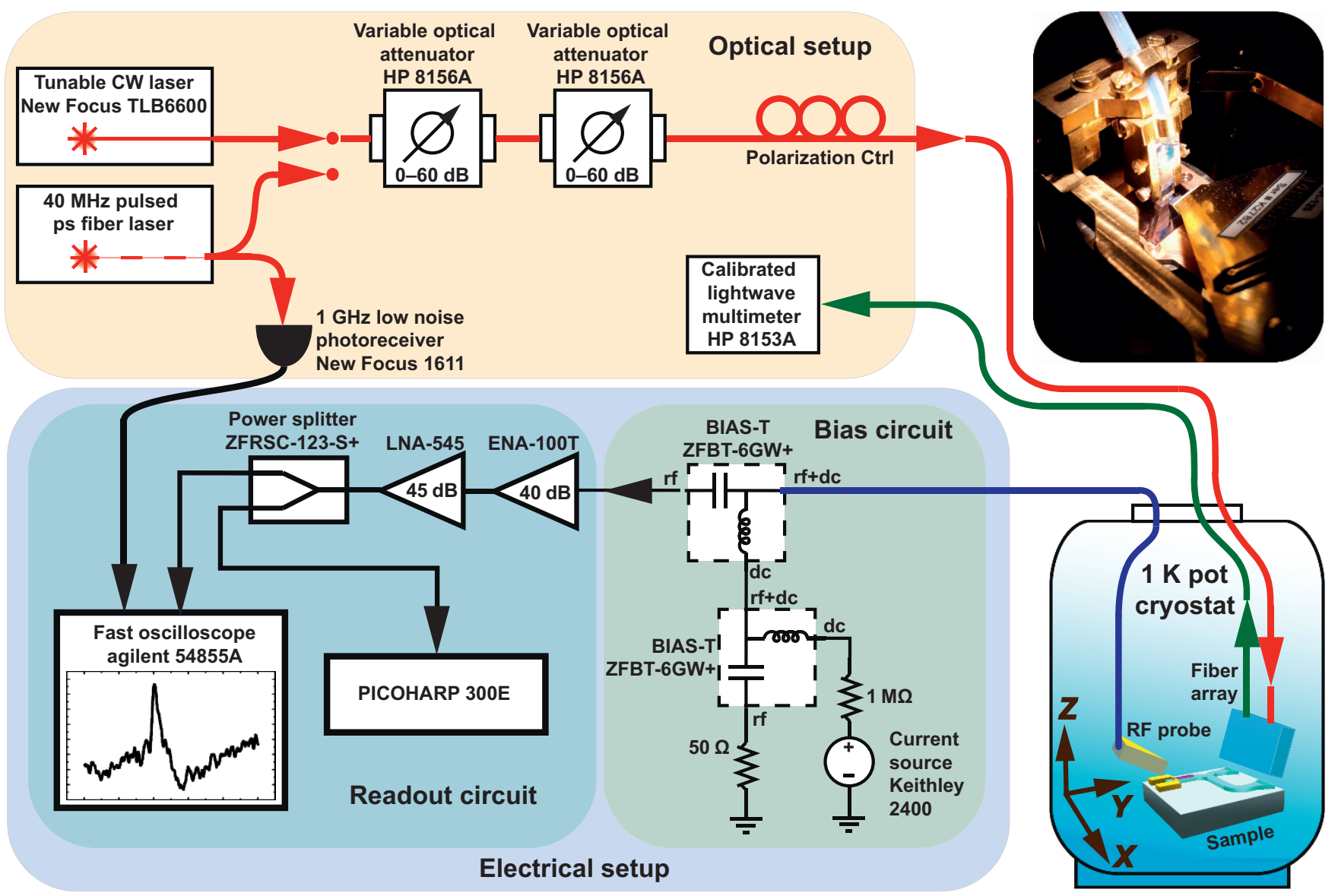

Figure 3 Cryogenic measurement setup. The detector sample is mounted inside the liquid helium cryostat at temperatures as low as $1 \mathrm{~K}$ and with optical access (red and green lines) and electrical access (blue line). The optical setup, including laser sources, attenuators and reference detectors, and the electrical setup, including the bias current source for the SNSPDs and the readout electronics, are operated at room temperature.

to a potential maximum detector count rate of approximately 200 $\mathrm{MHz}$, which compares favorably to the state-of-the-art photomultiplier tubes and avalanche photo-diodes ${ }^{13}$.

Determination of the on-chip detection efficiency

We then measure the on-chip detection efficiency (OCDE) of our detectors by comparing the photon flux arriving at the detector with the detector count rate in dependence of the bias current. The DCR is determined at the same certain bias current but with the laser turned off. Figure $4 \mathrm{~b}$ illustrates the detection efficiency measurement: each photonic circuit consists of an input grating coupler, which launches light from the fiber coupled laser into the diamond waveguide. After a subsequent 50:50 Y-splitter, half of the light is routed to the SNSPD, whereas the other half of the light is routed to an output grating coupler, which acts as a reference port. Considering the grating coupler, the 50:50 splitter and the propagation loss, the rate of photons arriving at the detector is determined. We then employ two optical attenuators to attenuate the light, such that the photon flux arriving at the detector is approximately $50000 \mathrm{~s}^{-1}$, which is far below the maximum detector count rate.

The OCDE is calculated from the measured count rate CR, corrected for the DCR, and the photon flux $\Phi$ arriving at the detector: $O C D E=\frac{C R-D C R}{\Phi}$. The photon flux is calculated using the on-chip reference port. For this, we first measure the transmitted power at the reference port $P_{\text {out }}$ for a known laser power $P_{\text {in }}$. The input and output coupling efficiency are considered to be the same. We calculate the coupling efficiency $C$ under the current measurement condition as $C=\sqrt{\frac{P_{\text {out }}}{P_{\text {in }} \times S \times W G L_{1}}}$, where $S=0.5$ is the splitting ratio of the Ysplitter and $W G L_{1}$ is the propagation loss along the waveguide connecting the input and output coupler.

To assess the propagation loss for half-etched diamond rib waveguides with $1 \mu \mathrm{m}$ widths, a chip with half-etched ring resonators is fabricated following the same fabrication steps as for the detector chip. For weakly coupled ring resonators with $1 \mu \mathrm{m}$ widths, the average quality factor accounts to $Q=9650$, which corresponds to a propagation loss of $\alpha=4.79 \mathrm{~dB} \mathrm{~mm}^{-1}$. The photon flux $\Phi$ arriving at the SNSPD is calculated as $\Phi=P_{\text {in,att }} \times C \times S \times W G L_{2}$, with the attenuated laser power $P_{\text {in,att }}$, coupling efficiency $C$ and the propagation loss $W G L_{2}$ along the waveguide connecting the Y-splitter and the SNSPD. The OCDE hence follows as $O C D E=\frac{C R-D C R}{P_{\text {in,att }} \times(C \times S) \times W G L_{2}}$

The count rate measurements are performed with an integration time of $2 \mathrm{~s}$. The uncertainty in the count rates is estimated from the variance of 10 consecutive measurements and (except for very low count rates) is found to be approximately $1 \%$. To account for statistical variations in both the grating coupler efficiency $C$ and the splitter ratio $S$, we evaluate the reference port transmissions $T=C^{2} \times S$ of the 

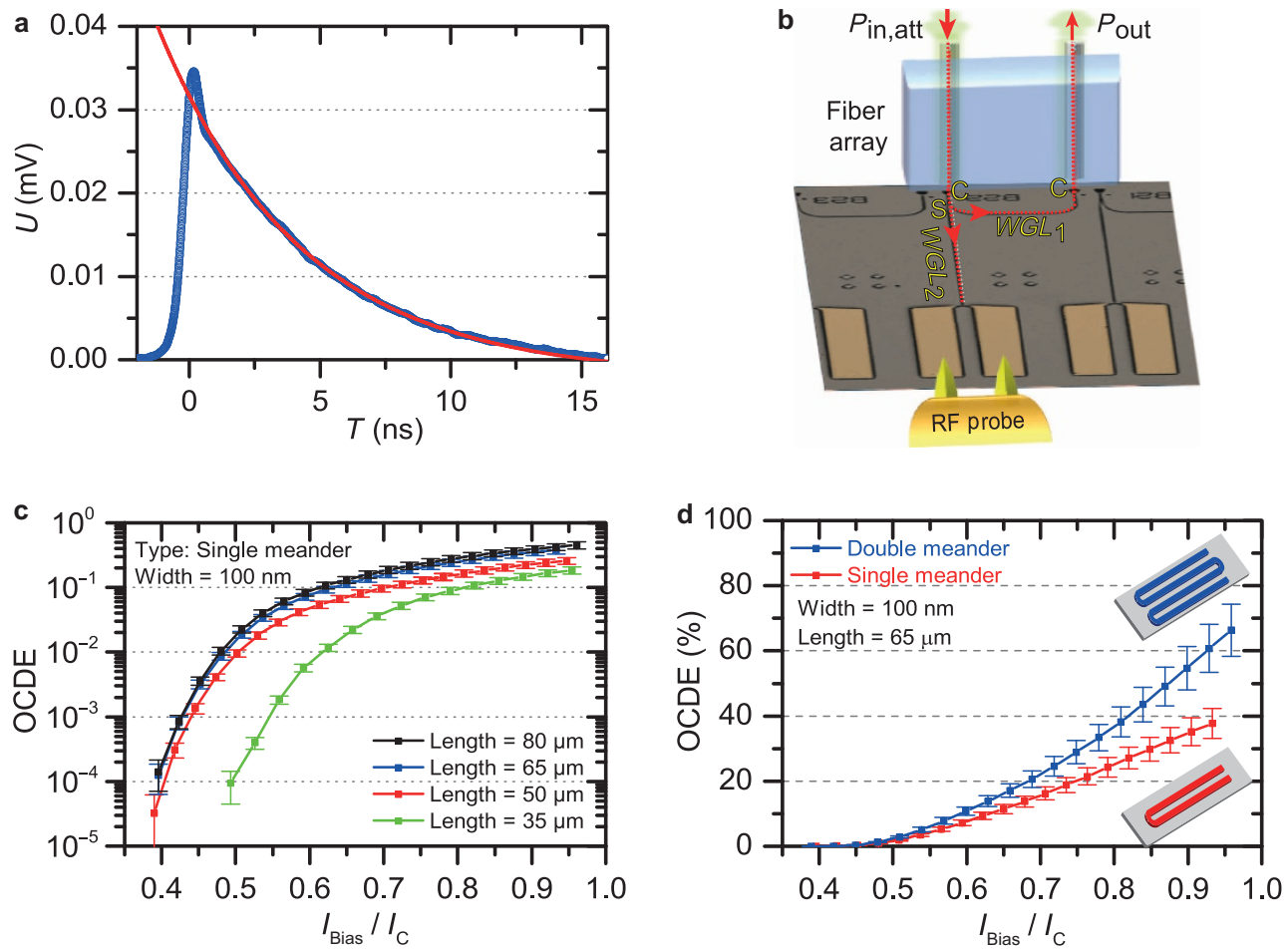

Figure 4 Characterization of the on-chip detection efficiency (OCDE). (a) Typical detected pulse shape (blue), resulting from the breakdown and the restoration of superconductivity following the absorption photon in a 35- $\mu \mathrm{m}$-long double meander. The presented data correspond to the average over 500 detection pulses. An exponential fit to the decay (red) reveals a decay time of $5.1 \mathrm{~ns}$. (b) Schematic showing the fiber array glass tip aligned to the input and reference grating coupler for photon flux monitoring and the electrical RF probe contacted to the metal contact pads of the NbN nanowire detector. (c) Dependence of the OCDE on the normalized bias current for 100-nm-wide single meander wires of varying length, measured at $1.9 \mathrm{~K}$. (d) Comparison of the OCDE for single and double meander detectors, measured at $1.9 \mathrm{~K}$, showing an increased OCDE up to $66 \%$.

balanced splitter devices shown in Figure 2a. The transmission of 54 devices of identical geometry is measured to be $T=3.44 \times 10^{-3} \pm$ $3.78 \times 10^{-4}$, which is equivalent to a relative variation in transmission of $\frac{\Delta T}{T}=\frac{\Delta\left(C^{2} \times S \times W G L_{1}\right)}{C^{2} \times S \times W G L_{1}}=10.98 \%$. For the OCDE calculation, one coupler and one splitter contribute to the photon flux. An upper bound for the uncertainty in $C \times S$ is hence given by $\frac{\Delta(C \times S)}{C \times S} \leq \frac{\Delta\left(C^{2} \times S \times W G L_{1}\right)}{C^{2} \times S \times W G L_{1}}=10.98 \%$. Eventually, the uncertainty of the propagation loss is calculated from the variation in quality factors extracted from the resonances of a ring resonator. For the studied ring resonators, we consistently observe a variation in the $Q$ factor of 5\%. Error propagation leads to a relative uncertainty in propagation loss of $\frac{\Delta W G L_{2}}{W G L_{2}}=1.57 \%$.

As expected, when increasing the bias current $I_{\text {bias }}$, the OCDE increases, as shown in Figure 4c. The bias current is given relative to the critical current of a nanowire. For a 100-nm-wide SNSPD, the measured critical current at $1.9 \mathrm{~K}$ is $3.4 \mu \mathrm{A}$, which corresponds to a critical current density of $j_{c}=0.74 \mathrm{MA} \mathrm{cm}^{-2}$. Several detectors with identical wire widths but increasing wire lengths from $35 \mu \mathrm{m}$ to $80 \mu \mathrm{m}$ are compared in this graph. For detectors with longer wires, the OCDE improves due to the increase in absorption efficiency ${ }^{51}$. In the case of an $80-\mu \mathrm{m}$-long single meander detector, the measured OCDE is $40 \%$ when it is biased at $96 \%$ of the critical current. As expected from the absorption measurements, detectors with the same device length but different meander conformations show different efficiencies (Figure 4d). Hence, the double meanders have a higher efficiency than do the single meanders. For a $65-\mu \mathrm{m}$-long double meander detector, the measured OCDE is $66 \%$. This means that of every 100 photons that arrive at the detector, on average, 66 detection events are recorded, which indicates that at the applied operation conditions, the energy of one IR photon is sufficient to break the superconductivity and may lead to a measureable output signal. Variations in the measured OCDE between devices of the same geometry are attributed to the residual surface roughness of the diamond layer, which can lead to constrictions in the wire, thus limiting the maximum possible bias current and hence the detector efficiency ${ }^{52}$. For the efficiency measurements, the photon flux arriving at the detector is set to be far below the saturation count rate of the SNSPDs. The detectors are operated at highly attenuated light intensities, where the incoming number of photons can be determined precisely such that the absorption of two photons in the same place and at the same time is highly improbable. In this regime, the SNSPDs operate as single-photon detectors. This is confirmed by observing that the measured count rate scales linearly with the input number of photons with a slope of one, when it is biased close to the critical current ${ }^{53,54}$. For this type of detector, this finding unambiguously demonstrates the single-photon detection capability $^{12}$.

\section{Determination of dark count rates and timing accuracy}

In addition to the OCDE, we analyze the DCR for every device using the same conditions that were used during the efficiency measurements. For this purpose, the laser is turned off, but the fiber array remains mounted on top of the grating couplers. Figure 5a shows the dark counts measured for the $65-\mu \mathrm{m}$-long double meander with 

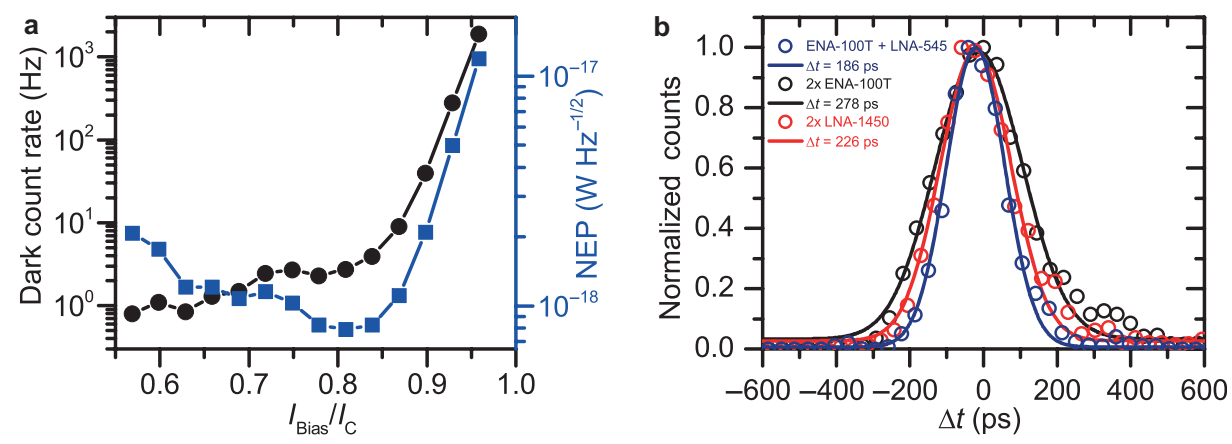

Figure 5 Dark count performance and timing resolution. (a) Dark count rate (black dots) and noise equivalent power (blue squares) measured at $1.9 \mathrm{~K}$ for the detector with $66 \%$ OCDE (blue data points in Figure 4d). The maximum dark count rate, when biased at $96 \%$ of the critical current, is $1.8 \mathrm{kHz}$. The minimum noise equivalent power, when biased at $81 \%$ of the critical current, amounts to $7.9 \times 10^{-19} \mathrm{~W} \mathrm{~Hz}^{-1 / 2}$. (b) Histograms of the SNSPD timing jitter measured with three different sets of amplifiers. Gaussian fits to the data reveal a FWHM jitter value of 186 ps for the best amplifier combination.

$66 \%$ OCDE (see Figure $4 \mathrm{~d}$ ). When increasing the bias current, the DCR rises exponentially ${ }^{55}$ to a maximum value of $1.8 \mathrm{kHz}$ when biased at $96 \%$ of the critical current. When the fiber array is mounted on top of the grating couplers, the DCR is typically limited by stray light, which is coupled into the cryostat via the optical fibers ${ }^{16}$. We calculate the on-chip noise equivalent power $\left(N E P_{\mathrm{OC}}\right)$, which is defined as $N E P_{\mathrm{OC}}=h v \times \frac{\sqrt{2 D C R}}{O C D E}$, where $h v$ is the photon energy. The lowest NEP for this device occurs when operating the detector at $81 \%$ of its critical current. The detector then shows an OCDE of 38\%, whereas the DCR is below $3 \mathrm{~Hz}$. The minimum noise equivalent power therefore amounts to $N E P_{\mathrm{OC}}=7.9 \times 10^{-19} \mathrm{~W} \mathrm{~Hz}^{-1 / 2}$.

We then analyze the timing accuracy of the SNSPDs by estimating their jitter contribution using a fast oscilloscope in histogram mode. A picosecond pulsed fiber laser at $1550 \mathrm{~nm}$ (PriTel Naperville, IL 60567-4025, USA) is used with a repetition rate of $40 \mathrm{MHz}$, providing $\sim 1$ ps pulses with a timing jitter below 1 ps. The laser light passes through a 50:50 splitter, and the resulting equal portions are routed to a fast low noise photo-receiver (1 GHz New Focus 1611) and to the SNSPDs in the cryostat. The SNSPDs are operated close to their critical current, and the detection events are recorded using a fast $6 \mathrm{GHz}$ digital oscilloscope (Agilent 54855A). The signal of the photo-receiver acts as the trigger signal. The jitter of the oscilloscope and the $1 \mathrm{GHz}$ photodetector have been measured to be less than 1 ps, respectively, well below the measured detection jitter. Therefore, the bandwidth limit and the total timing jitter are primarily determined by the low noise amplifiers and the SNSPD.

During the jitter measurements, the SNSPD signal is triggered at half the voltage pulse amplitude when the maximum slope is reached. The SNSPD is operated close to its critical current, and the measurement is performed using different sets of amplifiers, as shown in Figure 5b. Gaussian fits of the data reveal a FWHM jitter value of 186 ps. This value is strongly influenced by the electrical instrumentation, as shown in Figure 5b, and can be interpreted as an upper limit of the detector's true timing jitter.

\section{CONCLUSIONS}

Using the approach outlined above, we successfully show the realization of two key elements for on-chip quantum optics in diamond: efficient on-chip single-photon detectors combined with integrated photonic circuits. Our detectors are fast and have a decay time of 5.1 ns, which enables a maximum detector count rate up to $200 \mathrm{MHz}$. The devices also provide OCDEs as high as $66 \%$ and noise equivalent powers as low as $7.9 \times 10^{-19} \mathrm{~W} \mathrm{~Hz}^{-1 / 2}$. Because the OCDE improves for shorter wavelengths ${ }^{19,21,33}$, we expect that the detection efficiency for photons at visible wavelengths will be even higher and could be further increased with improved polishing procedures. Furthermore, the fabrication and design approach is general and can be directly transferred to single crystalline DOI substrates.

The implementation of traveling wave SNSPDs on diamond is a promising step toward a quantum-optics-on-a-chip platform that relies on monolithically joining single-photon sources, singlephoton routing and processing devices, as well as single-photon detectors. We envision that this could be achieved by combining optical cavities of high quality factor ${ }^{36,40,56-58}$ with color centers as single-photon sources and long waveguides for photon routing ${ }^{22,59}$. Furthermore, by utilizing the mechanical degrees of freedom that are available in free-standing diamond waveguides, both tunable elements and phase shifters ${ }^{28,29,60}$ can be obtained. By detecting these photons with fast and efficient single-photon detectors on the same chip, a full suite of functional elements is thus available for diamond quantum photonics.

\section{ACKNOWLEDGEMENTS}

Wolfram Pernice acknowledges support from the DFG (Grants Nos. PE 1832/ 1-1 \& PE 1832/2-1) and the Helmholtz Society (Grant No. HIRG-0005). We acknowledge support by Deutsche Forschungsgemeinschaft (DFG) and Open Access Publishing Fund of Karlsruhe Institute of Technology. Patrik Rath acknowledges financial support by the Deutsche Telekom Stiftung. The PhD education of Patrik Rath and Oliver Kahl is embedded in the Karlsruhe School of Optics \& Photonics (KSOP). We also acknowledge support by the DFG and the State of Baden-Württemberg through the DFG-Center for Functional Nanostructures (CFN) within subproject A6.4. We thank S. Kühn and S. Diewald for help with device fabrication and F. Pyatkov for help with initial resistivity measurements. The authors furthermore thank $\mathrm{F}$. Wu, M. Blaicher, and M. Stegmaier for helpful discussions concerning measurement setup and the manuscript. 
4 Vollmer F, Arnold S. Whispering-gallery-mode biosensing: label-free detection down to single molecules. Nat Methods 2008; 5: 591-596.

5 O'Brien JL, Furusawa A, Vučković J. Photonic quantum technologies. Nat Photon 2009; 3: 687-695.

6 Duan LM, Kimble HJ. Scalable photonic quantum computation through cavityassisted interactions. Phys Rev Lett 2004; 92: 127902.

7 Knill E, Laflamme R, Milburn GJ. A scheme for efficient quantum computation with linear optics. Nature 2001; 409: 46-52.

8 Kok P, Nemoto K, Ralph TC, Dowling JP, Milburn GJ. Linear optical quantum computing with photonic qubits. Rev Mod Phys 2007; 79: 135-174.

9 O'Brien JL. Optical quantum computing. Science 2007; 318: 1567-1570.

10 Dautet H, Deschamps P, Dion B, Macgregor AD, Macsween D et al. Photon counting techniques with silicon avalanche photodiodes. App/ Opt 1993; 32: 3894-3900.

11 Itzler MA, Jiang X, Entwistle M, Slomkowski K, Tosi A et al. Advances in InGaAsPbased avalanche diode single photon detectors. J Mod Opt 2011; 58: 174-200.

12 Gol'tsman GN, Okunev O, Chulkova G, Lipatov A, Semenov A et al. Picosecond superconducting single-photon optical detector. App/ Phys Lett 2001; 79: 705.

13 Hadfield $\mathrm{RH}$. Single-photon detectors for optical quantum information applications. Nat Photon 2009; 3: 696-705.

14 Natarajan CM, Tanner MG, Hadfield RH. Superconducting nanowire single-photon detectors: physics and applications. Supercond Sci Technol 2012; 25: 063001.

15 Pernice WHP, Schuck C, Minaeva O, Li M, Goltsman GN et al. High-speed and highefficiency travelling wave single-photon detectors embedded in nanophotonic circuits. Nat Commun 2012; 3: 1325

16 Schuck C, Pernice WHP, Tang HX. Waveguide integrated low noise NbTiN nanowire single-photon detectors with milli-Hz dark count rate. Sci Rep 2013; 3: 1893.

17 Schuck C, Pernice WHP, Ma X, Tang HX. Optical time domain reflectometry with low noise waveguide-coupled superconducting nanowire single-photon detectors. Appl Phys Lett 2013; 102: 191104.

18 Henrich D. Influence of Material and Geometry on the Performance of Superconducting Nanowire Single-Photon Detectors. Karlsruhe: KIT Scientific Publishing; 2013.

19 Marsili F, Bellei F, Najafi F, Dane AE, Dauler EA et al. Efficient single photon detection from $500 \mathrm{~nm}$ to $5 \mu \mathrm{m}$ wavelength. Nano Lett 2012; 12: 4799-4804.

20 Marsili F, Verma VB, Stern JA, Harrington S, Lita AE et al. Detecting single infrared photons with 93\% system efficiency. Nat Photon 2013; 7: 210-214.

21 Verevkin A, Zhang J, Sobolewski R, Lipatov A, Okunev 0 et al. Detection efficiency of large-active-area $\mathrm{NbN}$ single-photon superconducting detectors in the ultraviolet to near-infrared range. Appl Phys Lett 2002; 80: 4687.

22 Rath P, Gruhler N, Khasminskaya S, Nebel C, Wild C et al. Waferscale nanophotonic circuits made from diamond-on-insulator substrates. Opt Express 2013; 21: 11031.

23 Checoury X, Néel D, Boucaud P, Gesset C, Girard H et al. Nanocrystalline diamond photonics platform with high quality factor photonic crystal cavities. App/ Phys Lett 2012; 101: 171115.

24 Kolkowitz S, Jayich AC, Unterreithmeier QP, Bennett SD, Rabl P et al. Coherent sensing of a mechanical resonator with a single-spin qubit. Science 2012; 335: 1603-1606.

25 Neumann P, Jakobi I, Dolde F, Burk C, Reuter R et al. High precision nano scale temperature sensing using single defects in diamond. Nano Lett 2013; 13: 27382742.

26 Cai J, Jelezko F, Plenio MB. Hybrid sensors based on colour centres in diamond and piezoactive layers. Nat Commun 2014; 5: 4065.

27 Teissier J, Barfuss A, Appel P, Neu E, Maletinsky P. Strain coupling of a nitrogenvacancy center spin to a diamond mechanical oscillator. Phys Rev Lett 2014; 113: 020503.

28 Rath P, Khasminskaya S, Nebel C, Wild C, Pernice WHP. Diamond-integrated optomechanical circuits. Nat Commun 2013; 4: 1690.

29 Rath P, Ummethala S, Diewald S, Lewes-Malandrakis G, Brink D et al. Diamond electro-optomechanical resonators integrated in nanophotonic circuits. App/ Phys Lett 2014; 105: 251102.

30 Dolde F, Jakobi I, Naydenov B, Zhao N, Pezzagna S et al. Room-temperature entanglement between single defect spins in diamond. Nat Phys 2013: 9: 139-143.

31 Rogers LJ, Jahnke KD, Teraji T, Marseglia L, Müller C et al. Multiple intrinsically identical single-photon emitters in the solid state. Nat Commun 2014; 5: 4739.

32 Aharonovich I, Neu E. Diamond nanophotonics. Adv Opt Mater 2014; 2: 911-928.

33 Schuck C, Pernice WHP, Tang HX. NbTiN superconducting nanowire detectors for visible and telecom wavelengths single photon counting on Si3N4 photonic circuits. Appl Phys Lett 2013; 102: 051101.

34 Englund D, Shields B, Rivoire K, Hatami F, Vučković J et al. Deterministic coupling of a single nitrogen vacancy center to a photonic crystal cavity. Nano Lett 2010; 10: 3922-3926.

35 Faraon A, Santori C, Huang Z, Acosta V, Beausoleil R. Coupling of nitrogen-vacancy centers to photonic crystal cavities in monocrystalline diamond. Phys Rev Lett 2012; 109: 033604
36 Hausmann BJM, Shields BJ, Quan Q, Chu Y, de Leon NP et al. Coupling of NV centers to photonic crystal nanobeams in diamond. Nano Lett 2013; 13: 5791-5796.

37 Doherty MW, Manson NB, Delaney P, Jelezko F, Wrachtrup J et al. The nitrogenvacancy colour centre in diamond. Phys Rep 2013; 528: 1-45.

38 Lee JC, Aharonovich I, Magyar AP, Rol F, Hu EL. Coupling of silicon-vacancy centers to a single crystal diamond cavity. Opt Express 2012; 20: 8891-8897.

39 Neu E, Hepp C, Hauschild M, Gsell S, Fischer M et al. Low-temperature investigations of single silicon vacancy colour centres in diamond. New J Phys 2013; 15: 043005.

40 Riedrich-Möller J, Arend C, Pauly C, Mucklich F, Fischer M et al. Deterministic coupling of a single silicon-vacancy color center to a photonic crystal cavity in diamond. Nano Lett 2014; 14: 5281-5287.

41 Taillaert D, Bogaerts W, Bienstman P, Krauss TF, Van Daele P et al. An out-of-plane grating coupler for efficient butt-coupling between compact planar waveguides and single-mode fibers. IEEE J Quant Electron 2002; 38: 949-955.

42 Rath P, Khasminskaya S, Nebel C, Wild C, Pernice WHP. Grating-assisted coupling to nanophotonic circuits in microcrystalline diamond thin films. Beilstein J Nanotechnol 2013; 4: 300-305.

43 Füner M, Wild C, Koidl P. Novel microwave plasma reactor for diamond synthesis. App/ Phys Lett 1998; 72: 1149

44 Füner M, Wild C, Koidl P. Simulation and development of optimized microwave plasma reactors for diamond deposition. Surf Coat Technol 1999, 116/ 119: 853-862.

45 Thomas ELH, Nelson GW, Mandal S, Foord JS, Williams OA. Chemical mechanical polishing of thin film diamond. Carbon 2014; 68: 473-479.

46 Ummethala S, Rath P, Lewes-Malandrakis G, Brink D, Nebel C et al. High-Q optomechanical circuits made from polished nanocrystalline diamond thin films. Diamond Relat Mater 2014; 44: 49-53.

47 Rath P, Hirtz M, Lewes-Malandrakis G, Brink D, Nebel C et al. Diamond nanophotonic circuits functionalized by dip-pen nanolithography. Adv Opt Mater 2015; 3: 328335.

48 Ovartchaiyapong P, Pascal LMA, Myers BA, Lauria P, Bleszynski Jayich AC. High quality factor single-crystal diamond mechanical resonators. Appl Phys Lett 2012; 101: 163505.

49 Tao Y, Degen C. Facile fabrication of single-crystal-diamond nanostructures with ultrahigh aspect ratio. Adv Mater 2013; 25: 3962-3967.

50 Lee JC, Magyar AP, Bracher DO, Aharonovich I, Hu EL. Fabrication of thin diamond membranes for photonic applications. Diamond Relat Mater 2013; 33: 45-48.

51 Kovalyuk V, Hartmann W, Kahl O, Kaurova N, Korneev A et al. Absorption engineering of $\mathrm{NbN}$ nanowires deposited on silicon nitride nanophotonic circuits. Opt Express 2013; $21: 22683$.

52 Kerman AJ, Dauler EA, Yang JK, Rosfjord KM, Anant V et al. Constriction-limited detection efficiency of superconducting nanowire single-photon detectors. App Phys Lett 2007; 90: 101110.

53 Elezov MS, Semenov AV, An PP, Tarkhov MA, Goltsman GN et al. Investigating the detection regimes of a superconducting single-photon detector. J Opt Technol 2013 , 80: 435 .

54 Ferrari S, Kahl O, Kovalyuk V, Goltsman GN, Korneev A et al. Waveguide-integrated single- and multi-photon detection at telecom wavelengths using superconducting nanowires. Appl Phys Lett 2015; 106: 151101.

55 Kitaygorsky J, Zhang J, Verevkin A, Sergeev A, Korneev A et al. Origin of dark counts in nanostructured NbN single-photon detectors. IEEE Trans App/ Supercond 2005, 15: 545-548.

56 Bayn I, Mouradian S, Li L, Goldstein JA, Schröder T et al. Fabrication of triangular nanobeam waveguide networks in bulk diamond using single-crystal silicon hard masks. Appl Phys Lett 2014; 105: 211101.

57 Hausmann BJM, Bulu I, Deotare PB, McCutcheon M, Venkataraman V et al. Integrated high quality factor optical resonators in diamond. Nano Lett 2013; 13: 1898-1902.

58 Lee JC, Bracher DO, Cui S, Ohno K, Mclellan CA et al. Deterministic coupling of deltadoped nitrogen vacancy centers to a nanobeam photonic crystal cavity. App I Phys Lett 2014; 105: 261101.

59 Hiscocks MP, Ganesan K, Gibson BC, Huntington ST, Ladouceur F et al. Diamond waveguides fabricated by reactive ion etching. Opt Express 2008; 16: 19512 19519

60 Sohn YI, Burek MJ, Lončar M. Dynamic actuation of single-crystal diamond nanobeams 2014; arXiv preprint:1408.5822.

cc)(i) (2) This work is licensed under a Creative Commons AttributionBY NG SA NonCommercial-ShareAlike 3.0 Unported License. The images or other third party material in this article are included in the article's Creative Commons license, unless indicated otherwise in the credit line; if the material is not included under the Creative Commons license, users will need to obtain permission from the license holder to reproduce the material. To view a copy of this license, visit http://creativecommons.org/licenses/by-nc-sa/4.0/ 